\title{
Review of: "Microtubule deacetylation reduces cell stiffness to allow the onset of collective cell migration in vivo"
}

\author{
Paul Sundaram ${ }^{1}$ \\ 1 University of Puerto Rico
}

Potential competing interests: The author(s) declared that no potential competing interests exist.

Interesting article on the dynamic stiffness of Neural Crest (NC) cells as requirement for Collective Cell Migration (CCM). The authors have carried out various tests on NC cells in Xenopus laevis embryos as well as simulations using a mathematical model to determine $\mathrm{Rg}$ (radius of gyration) as a measure of CCM. The authors have demonstrated, starting with a number of theses, how cells migrate in their "dynamic and convoluted" in vivo environment by adjusting their mechanical properties in relation to the substrate. Some questions:

1. How is CCM measured in vivo? How many cells are tracked to use a threshold value to determine that CCM has taken place?

2. Line 88/89: do you start measuring elastic modulus of the cells and the mesoderm at regular intervals? Does this value then decrease gradually or suddenly at which point CCM occurs? Is this process reversible? Can you stop CCM while it is occurring by changing the elastic modulus of the cells?

3. It appears that the stability of NC cells in the tissue depends on substrate stiffness and not cell stiffness? Do cells respond to substrate stiffness by altering their elastic modulus? Lines 97-99 seem a little contradictory. Are cells which normally grow on a specific substrate tissue always stiffer that the substrate?

4. Have the authors tested/will test cells other than NC cells to put forth their theses, even in silico?

5. Authors mention that $E(\operatorname{sub}) / E(i)$ is low $(<0.22)$ for non-migratory behavior or high $(\sim 1)$ for migratory behavior. What about intermediate values? When do cells become totally non-migratory? Does this implicate hyperacetylation of microtubules? Thus, are there limits in $E(s u b) / E(i)$ values depending on cell type in which range cells show CCM?

6. Is cell stiffening by MT acetylation a reversible process? Can the degree of MT acetylation be linearly correlated with length of MTs and hence the elastic stiffness of such cells? Are there other mechanosensory cues for migratory behavior in cells?

7. This article brings together much information worthy of further avenues of research. The authors have convincingly proved their theses, although some issues must still be ironed out. 
